\title{
Body building is about teamwork
}

DOI:

10.1038/nrc2038

\section{URLs}

$\mathrm{RB}$

http://www.ncbi.nlm.nih.gov/ entrez/query.fcgi? $\mathrm{db}=$ gene\&c $\mathrm{md}=$ RetrieveEdopt=full_ report\&list_uids $=5925$

P53

http://www.ncbi.nlm.nih.gov/ entrez/query.fcgi?db=gene\&c $\mathrm{md}=$ Retrieve\&dopt=full report\&list_uids $=7157$

P63

http://www.ncbi.nlm.nih.gov/ entrez/query.fcgi?db=gene\&c $\mathrm{md}=$ Retrieve\&dopt=full report\&list_uids $=8626$

P73

http://www.ncbi.nlm.nih.gov/ entrez/query.fcgi?db=gene\&c $\mathrm{md}=$ Retrieve\&dopt=full report\&list_uids=7161

Rhabdomyosarcoma http://www.cancer.gov/ cancertopics/types/ childrhabdomyosarcoma
The tumour suppressor p53 is deregulated in many human cancers, but exactly how p53 cooperates with its family members, p63 and p73, to control tumour formation remains unclear. Thorsten Stiewe and colleagues show that the family members act differently, but synergistically, on the retinoblastoma protein (RB) during myogenic differentiation, and that their loss of function promotes malignant transformation.

To investigate the role of the p53 family members in myogenesis, Stiewe and colleagues used C2C12 myoblasts, which faithfully mimic skeletal muscle differentiation in vitro, transduced with $\Delta \mathrm{Np} 73 \alpha$, a $\mathrm{p} 73$ isoform that functions as a transdominant inhibitor of all the p 53 family members. $\triangle \mathrm{Np} 73 \alpha$ interfered with the formation of differentiated myotubes and prevented the permanent withdrawal of $\mathrm{C} 2 \mathrm{C} 12$ cells from the cell cycle. Genome-wide expression analysis showed that $\mathrm{C} 2 \mathrm{C} 12-\Delta \mathrm{Np} 73 \alpha$ myoblasts had an expression pattern similar to that seen in RB-deficient myoblasts, so could a defect in RB activation be the underlying cause of the differentiation block?

The activation of $\mathrm{RB}$ was significantly reduced in $\mathrm{C} 2 \mathrm{C} 12-\Delta \mathrm{Np} 73 a$ myoblasts, and high levels of inactive hyperphosphorylated RB were evident. Ectopic expression of a constitutively active mutant of RB, but not wild-type $\mathrm{RB}$, could restore differentiation ability in the $\mathrm{C} 2 \mathrm{C} 12-\Delta \mathrm{Np} 73 a$ myoblasts. Moreover, ectopic expression of wild-type RB together with p57 (also known as CDKN1C, which prevents the phosphorylation and inactivation of $\mathrm{RB}$ ) could also rescue the differentiation block. But how are the individual $\mathrm{p} 53$ proteins involved in the activation of RB?
Dominant-negative inhibitors of either p53 (p53DD) or p63 and p73 (p73DD) significantly reduced the differentiation ability of $\mathrm{C} 2 \mathrm{C} 12$ myoblasts, and when expressed together they caused a further reduction in differentiation, indicating complementary functions in the control of differentiation. RB expression was inhibited by p53DD, whereas p57 expression was impaired by $\mathrm{p} 73 \mathrm{DD}$. Chromatin immunoprecipitation confirmed that, in differentiating myoblasts, p53 binds the $R b 1$ promoter, and p63 and TAp73 (another p73 isoform) bind the Cdkn1c promoter. Stiewe and colleagues conclude that for efficient myogenic differentiation, p53 regulates $\mathrm{RB}$ expression whereas p63 and p73 control, through p57, the phosphorylation of RB.

Differentiation failure is a characteristic feature of rhabdomyosarcoma (RMS). C2C12- $\Delta \mathrm{Np} 73 a$ myoblasts did not form tumours in nude mice, indicating that the inhibition of differentiation is not sufficient for tumour formation. However, $\triangle \mathrm{Np} 73 \mathrm{a}$ can cooperate with the known RMS oncoproteins insulin-like growth factor 2 (IGF2) and PAX3-FKHR to induce transformation. Both IGF $2+\Delta \mathrm{Np} 73 a$ and PAX3-FKHR $+\Delta$ Np73a-expressing $\mathrm{C} 2 \mathrm{C} 12$ myoblasts were tumorigenic in nude mice. Furthermore, reverse transcription-PCR confirmed aberrant high expression of $\mathrm{p} 73$ isoforms in both human RMS samples and RMS cell lines. In addition, in RMS cell lines, inhibition of $\Delta \mathrm{Np} 73 \alpha$ by RNA interference induced p57 and caused cell-cycle arrest.

The authors conclude that the induction of cellular differentiation contributes to the tumour-suppressor activities of the p53 family, and provides an explanation for the high frequency of $\mathrm{p} 53$ pathway alterations in RMS patients.

Rebecca Robey

ORIGINAL RESEARCH PAPER Cam, H. et al. p5

family members in myogenic differentiation and rhabdomyosarcoma development. Cancer Cell 10, 281-293 (2006)

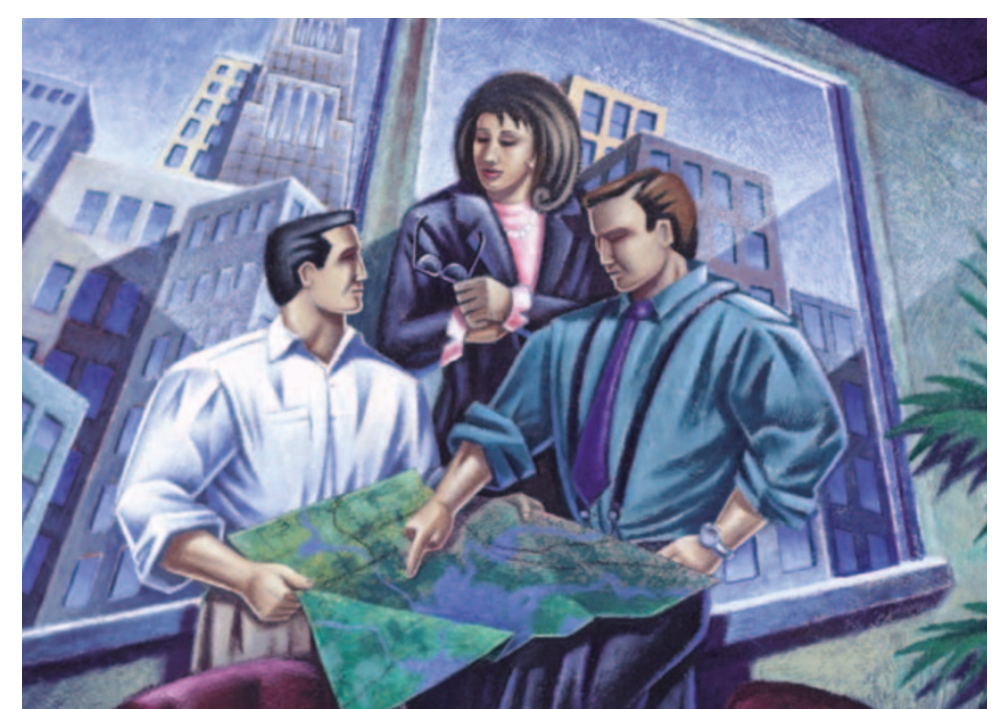

